$\xi=-1$

\title{
Design of SCADA based solar power plant for clean water irrigation system in remote areas
}

\author{
Rais Mu’ammar, Prapto Nugroho, Lesnanto Multa Putranto* \\ Department of Electrical and Information Technology Engineering, Universitas Gadjah Mada Yogyakarta, Indonesia \\ * Corresponding author: lesnanto@.ugm.ac.id
}

\begin{abstract}
The study aimed to design a supervisory control and data acquisition (SCADA) system to control solar power plants for clean water irrigation system during the dry season in remote areas. The SCADA system contrived is expected to be able to meet the main elements of the SCADA architecture. Accordingly, following steps were conducted, starting from designing the human machine interface (HMI) in the master station (MS), mapping out the microcontroller as a remote terminal unit (RTU) and designing the communication path between MS and RTU. Furthermore, the SCADA system was tested by the system test to evaluate the SCADA system that was designed meet minimum standard and running well. After tested, the result showed that the system is successfully designed to meet the main elements of SCADA architecture. The system is also able to monitor and control remotely, resulting in more efficient control of user work as well as rapid decision making in order to minimize damage potential when an interruption occurs. For both control and monitor, the MS and RTU in the SCADA system wirelessly communicate.
\end{abstract}

Keywords: SCADA architecture; MS; RTU; Communication lines; Solar power plant

\section{Introduction}

A geographically suitable supervisory control and data acquisition (SCADA) system is urgently needed particularly when implemented to remote areas. So far, implementation of SCADA system is limited due to several factors such as a large and complex system, expensive prince, and adequate source of electricity requirement. Designing the SCADA system for remote areas with economically feasible, specific system size, and easily controlled are considered to be more promising. This research developed and planned a SCADA system to control solar power plant in the clean water irrigation system in order to help villagers in remote areas which is less electricity can access clean water easily.

Prior to the problem, there are some report based on literature review showed the progress of research [1] - [10]. From these studies, general elements of SCADA architecture are explained. Regarding on the SCADA architecture, studies [1] - [4] showed that the SCADA system generally has 3 main important elements, including RTU as data collector from the field, MS as monitor and control, and communication line to the link between RTU and MS The RTU can be designed with a microcontroller as in research [1] and [2]. Prior to SCADA communication line system for solar power plant, wireless communication method is widely used as mentioned in the research [3], [4] and [5]. The research [3] used the xbee module as a wireless communication line. The advantage of Xbee module is able to operate in remote areas where there is no internet connectivity. Moreover, the MS side or graphic user interface (GUI) of the SCADA system, labview software can be used as HMI as in the study [6], [7], [8] and [9].

As report from several studies, the novelty of this research attempt to combine several research methods that already exist in the research [1] - [9]. The incorporation of several methods of research aims to ensure that the main elements of the SCADA architecture can be fulfilled. Supporting this, HMI was designed on the MS, including the microcontroller as a RTU and communication lines between MS and RTU. This study is successfully designed SCADA system that can effectively control and monitor to solar power plants, provide the desired information in the form of measurement quantities, reporting, historical logging and data recording. In addition, the system also effectively controls the system remotely to make it easier for users. Apart from that, it is also able to minimize user work, as well as saving time in the process of decision making in order to minimize damage to the system. To ensure that the system is running well, the SCADA system was tested. The test is divided into three parts, namely testing against HMI, RTU and communication lines. The HMI test is purposed to ensure the HMI in the monitor and control of the system. Testing the RTU includes data acquisition of each sensor and component control in the PLTS. Thereafter, the communication lines testing is conducted to provide information about the distance range between MS and RTU to communicate wirelessly.

\section{Design system}

The SCADA design system in this study was conducted in the three main elements both in terms of software and hardware. The first element is the design of MS which is focused on HMI, the second element is the design of the RTU and the third element is the design of communication lines. Apart from that, solar power plant system planning is also needed. This plan aims to determine the size of the solar power plants system that is controlled by the SCADA system so that the specification of the SCADA system can be determined. 


\subsection{Solar power plant system planning}

This step is aimed to plan the size of the solar power plant system based on predetermined equations. For instance, to calculate the daily load on the system, determine the amount and capacity of $\mathrm{PV}$, determine the amount and capacity of the battery, determine the capacity of the SCC, determine the capacity of the inverter based on research [10], determine the motor capacity and determine the capacity of water storage. Results from this calculation are used as data to the size of SCADA designed based on the size of the solar power plant.

1. Calculate the daily load on the system

The amount of daily load installed on the system can be calculated by using equation (1).

$$
E_{T}=\sum_{i}^{n} W_{i} \cdot h
$$

Where:

$\mathrm{E}_{\mathrm{T}}=$ The amount of power necessity $(\mathrm{Wh})$

$\mathrm{W}_{\mathrm{i}}=$ Nominal power for each load $\mathrm{i}(\mathrm{W})$

$\mathrm{h}=$ Duration of daily usage (hours)

Then enter the value of the system losses. The calculation can be done with equation (2).

$E_{R}=\frac{E_{T}}{R}$

Where:

$\mathrm{E}_{\mathrm{R}}=$ The amount of real power necessity $(\mathrm{Wh})$

$\mathrm{R}=$ Global electrical performance of the photovoltaic installation $(0.809)$

1. Determine the amount of PV

The amount of PV can be determined by the equation (3).

$N_{P V} \approx \frac{E_{R}}{W_{P} \cdot t_{h}}$

Where:

$\mathrm{N}_{\mathrm{PV}}=$ The amount of necessity if PV

$\mathrm{W}_{\mathrm{p}} \quad=$ Maximum power potential of $\mathrm{PV}(\mathrm{Wp})$

$\mathrm{t}_{\mathrm{h}} \quad=$ Lighting time per day (hours/ day)

2. Determine the capacity and number of battery

Battery capacity can be determined by the equation (4).

$C_{\text {batt }}=\frac{E_{R}}{V_{\text {batt }} \cdot D_{d}}$

Where:

$\mathrm{C}_{\text {batt }}=$ Battery capacity $(\mathrm{Ah})$

$\mathrm{V}_{\text {batt }}=$ Battery voltage $(\mathrm{V})$

$\mathrm{D}_{\mathrm{d}}=$ deep of discharge $(\%)$

Following this, the number of battery is calculated by equation (5).

$N_{\text {batt }} \approx \frac{C_{\text {batt }}}{C_{\text {ref }}}$

Where:

$\mathrm{N}_{\text {batt }}=$ Number battery used

$\mathrm{C}_{\text {batt }}=$ Battery capacity $(\mathrm{Ah})$

$\mathrm{C}_{\text {ref }}=$ Reference battery capacity $(\mathrm{Ah})$
3. Determine the SCC capacity

The SCC capacity determines by equation (6).

$I_{\text {maks }}=I_{S c} \cdot N_{P V}$

Where:

$\mathrm{I}_{\text {maks }}=$ Current amount on the system $(\mathrm{A})$

$\mathrm{I}_{\mathrm{sc}}=$ Short circuit current $(\mathrm{A})$

$\mathrm{NPV}=$ Number of PV used

4. Determine the inverter capacity

Interver capacity determines by the equation (7).

$P_{i n v} \approx P_{S}$

Where:

$\mathrm{P}_{\text {inv }}=$ Power inverter $(\mathrm{W})$

$\mathrm{P}_{\mathrm{s}}=$ The total load used (W)

5. Determine motor specifications

Motor specification can be determined by equation (8).

$Q_{m}=\frac{q_{d} \cdot p_{w}}{t_{h}}$

Where:

$\mathrm{Q}_{\mathrm{m}}=$ water debit per minute (Liter/ minute)

$\mathrm{q}_{\mathrm{d}}=$ Standard for villagers need of water (liter/ person/ day)

$\mathrm{p}_{\mathrm{w}}=$ Number of villagers

$\mathrm{t}_{\mathrm{h}}=$ Motor works duration (minute)

In addition, to know the amount of electrical power needed by the motor per day, equation (9) will be used

$P_{m}=\left(\frac{\left(\frac{q_{d} \cdot p_{w}}{Q_{m}} P_{r}\right)}{60}\right)$

Where:

$\mathrm{P}_{\mathrm{m}}=$ The amount of power needed $(\mathrm{Wh})$

$\mathrm{P}_{\mathrm{r}}=$ Power shown on the motor $(\mathrm{W})$

The results of the solar power plant system planning will be used as data to determine the size of the SCADA system designed.

\subsection{Design of the SCADA system}

The design for SCADA system is illustrated in Fig. 1 which is consisting of MS, RTU, and connecting communication lines between MS and RTU. The SCADA system design in this research consists of three main elements both for software and hardware. The first element is MS design that focuses on HMI, the second element is on RTU design, and the third element is designed for communication lines. The MS of the system functions to control and monitor the controlled system. Monitoring is conducted by the power produced by PV, the battery used, the inverter output, the working pump motor, and the amount of water in the storage. The system then provides the information needed in the form of measurement quantities, reporting, historical data and recording historical data. The saved data can be displayed again to find out the data recording process. The contents of the recorded data are the time of data collection, the electrical quantities and the condition of the equipment. For control purpose, the system is controlling remotely both for battery and motor control. The MS is also equipped with an alarm, if the condition of the equipment is limited to a predetermined threshold, alarm works.

The second element is RTU that functions as the data collector from the field and send it to MS. The MS will command the RTU 
to control the equipment around. The microcontroller used to build RTU in this study is the Atmega 328 microcontroller. The 328 microcontroller has six analog pins which are used for sensor pins, then 14 digital pins for 8 channel relay modules. This microcontroller has a clock speed of $16 \mathrm{MHz}$. This initial size speed is expecting to be able to quickly sampling the data for sensors.

The design of communication lines to connect RTU and MS is conducted for data exchange purposes. The role of RTU is sending the data to the MS by communication lines, vice versa. For communication lines device, this study uses Xbee S2C. Xbee S2C is an electronic device that functions as a data sender and receiver wirelessly. Xbee S2C is standardized IEEE protocol for 802.15.4 protocol.

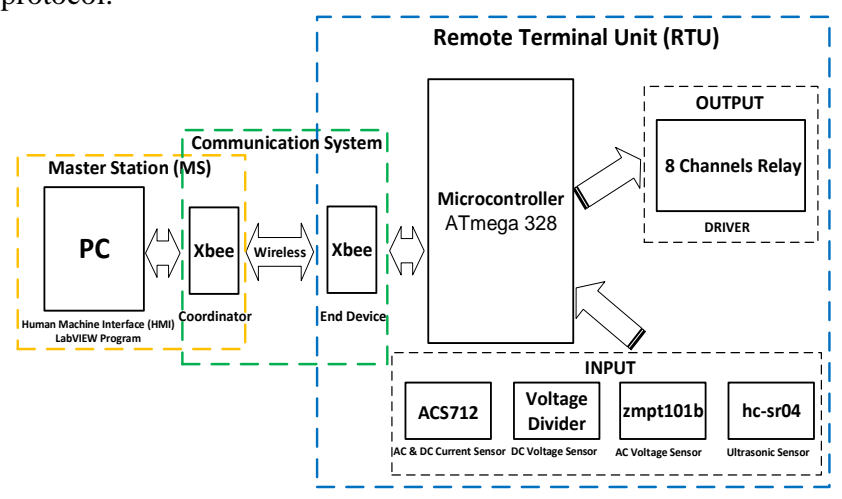

Fig. 1: Design of SCADA system

The RTU installation on the solar power plant for clean water irrigation system shows in Fig. 2. The designed RTU is equipped with sensor and driver modules. These sensor modules are comprised of four types of sensor modules, namely DC sensor modules, AC voltage sensor modules, DC and AC current sensor modules, and ultrasonic sensor modules. The DC voltage sensor module is used to detect DC voltage in PV and battery while the $\mathrm{AC}$ voltage sensor module is used to detect the $\mathrm{AC}$ voltage on the inverter and motor. Further, the DC and AC current sensor modules are used to detect the DC currents in PV and detect AC currents on the inverter and motor, respectively. To detect water level, the ultrasonic sensor module is used prior to water storage. In addition to the sensor modules, there are a drivers using 8 channel relay modules. This driver functions to control PV, batteries and motors.

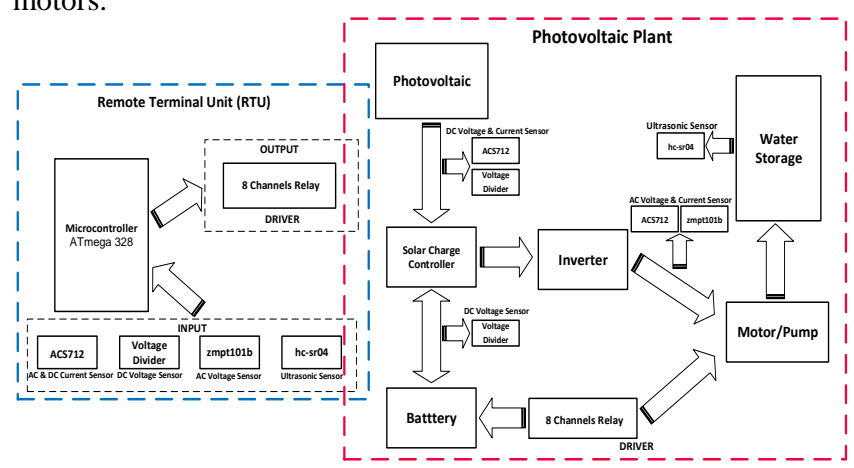

Fig. 2: The RTU installation in the solar power plant system

In this study, the solar power plant installation components consisted of PV, SCC, batteries, inverters, motors and water storage. PV serves as an alternative energy producer while the SCC functions as a controller and limits the electrical energy charged to the battery. The battery's role is to store the energy for PV and load suppliers then the inverter plays as a converter of DC electricity from the battery to the $\mathrm{AC}$ which is connected to the motor. Furthermore, water is transported into the water storage by motor. In this condition, water storage functions as a temporary water reservoir.

\subsubsection{Design of MS as HMI}

The purpose of design the HMI is primarily to process and display the data sent by the RTU. Design is also required to simplify data saving by the user and to know the status of the equipment element condition as indicated by the ON/ OFF indicatior and alarm. The user is also facilitated to remotely control the PV, battery, and motor. Apart from that, HMI also provides convenience to users in navigating, minimizing user's work, helping to make rapid decision making in order to minimize problems that potentially occur when there is a disruption to the system. After the design of HMI is finished, the list of features that have been implemented into this SCADA system can be observed in the Tab. 2 .

\subsubsection{Design of RTU}

The designed RTU is equipped with sensor modules and driver modules. These sensor modules consist of DC voltage sensor modules, AC voltage sensor modules, DC and AC current sensor modules, and ultrasonic sensor modules.

1. DC voltage sensor module

DC voltage sensor is used to detect DC voltage in PV and batteries. The DC voltage sensor schematic is provided in Fig. 3.

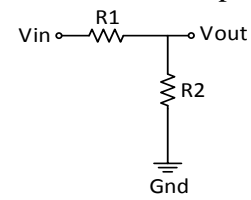

Fig. 3: The schematic of DC voltage sensor

The DC voltage sensor serves as a voltage divider circuit, then applies to equation (10).

$$
\text { Vout }=\operatorname{Vin} \frac{R 2}{R 1+R 2}
$$

Where:

$\mathrm{V}_{\text {out }} \quad=$ output voltage $(\mathrm{V})$

$\mathrm{V}_{\text {in }} \quad=$ input voltage $(\mathrm{V})$

$\mathrm{R} 1, \mathrm{R} 2$ = value for resistor 1 and resistor $2(\Omega)$

\section{The AC voltage sensor module}

The AC voltage sensor module is used to detect $\mathrm{AC}$ voltage on the inverter and motor. The schematic of this module is provided in Fig. 4.

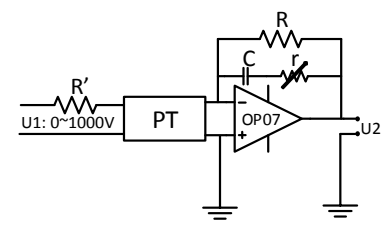

Fig. 4: The schematic of AC voltage sensor

It is assumed that the voltage supply is a sinusoidal perfection, therefore the effective voltage can be obtained from the peak voltage using Equation (11)[11].

$V_{\text {rms }}=\frac{V_{\text {peak }}}{\sqrt{2}}=\frac{V_{\text {peak to peak }}}{2 \sqrt{2}}$

Where:

$\mathrm{V}_{\mathrm{rms}} \quad=$ Effective voltage $(\mathrm{V})$

$\mathrm{V}_{\text {peak }} \quad=$ Peak voltage $(\mathrm{V})$

$\mathrm{V}_{\text {peak to peak }}=$ Peak to peak voltage $(\mathrm{V})$ 
3. DC and AC current sensor modules

The DC and AC current sensor module is used to detect DC current at PV and battery, and AC current at the inverter and motor. The schematic of this module is provided in Fig. 5.

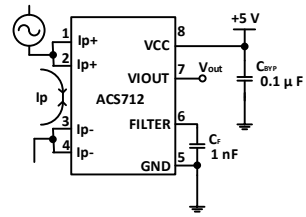

Fig. 5: The schematic of DC and AC [12]

4. Ultrasonic sensor module

The ultrasonic sensor module is used to detect water level in the water storage. This module work principle can be seen in Fig. 6 .

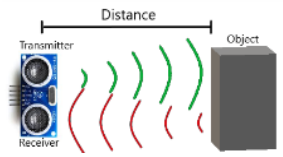

Fig. 6: Work principle of ultrasonic module

Wave travel time when osculate the object and come in back to the receiver is the result of distance converted as the following equation (12) applies.

$$
s=v \frac{t}{2}
$$

where:

$\mathrm{s}=$ Distance $(\mathrm{cm})$

$\mathrm{v}=$ Velocity $(343 \mathrm{~m} / \mathrm{s})$

$\mathrm{t}=$ Time $(\mathrm{s})$

\section{Driver module}

The driver module is used for several purposes, including connecting process the PV to the system, controlling the battery and motor. The schematic of the driver module can be seen in Fig. 7

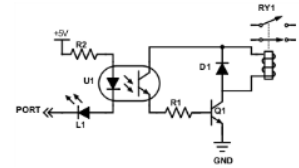

Fig. 7: Schematic of driver module

\subsubsection{Design of communication lines}

Design of communication line aims to confirm the communication line between MS and RTU. Configuration is conducted by setting xbee module as coordinator and end devices equipped with 802.15.4 IEEE standardized protocol. Xbee module configuration as coordinator and end device is required in order to make xbee modules able to receive and send data in two directions path. The important parameters should be set are available in Tab. 1

Table 1: Setting Xbee as Coordinator and End Devices

\begin{tabular}{|c|c|c|}
\hline No & Parameters & Setting \\
\hline 1 & Protocol & 802.15 .4 \\
\hline 2 & ID PAN ID & 12345 \\
\hline 3 & CE coordinator & Coordinator/end devices \\
\hline 4 & Baud rate & 9600 \\
\hline
\end{tabular}

\section{Result and discussion}

The results of this SCADA system planning are provided in Tab. 2. The assumption is used at load usage of 75 watts and water consumption of 100 Liters/ day/ person in total population of 100 people. The size of SCADA system is determined based on the result of the solar power plant system.

Table 2: Results of solar power plant (SPP) and SCADA systems planning

\begin{tabular}{|c|c|c|c|c|}
\hline No & $\begin{array}{l}\text { SPP com- } \\
\text { ponents }\end{array}$ & $\begin{array}{l}\text { Equation im- } \\
\text { plementation } \\
(1)-(9)\end{array}$ & $\begin{array}{l}\text { SCADA fea- } \\
\text { tures }\end{array}$ & RTU \\
\hline 1 & PV & $100 \mathrm{Wp}$ & $\begin{array}{l}\text { PV power } \\
\text { Monitor }\end{array}$ & $\begin{array}{c}\text { Microcontroller } \\
\text { ATmega } 328\end{array}$ \\
\hline 2 & SCC & $10 \mathrm{~A}$ & $\begin{array}{c}\text { Battery ca- } \\
\text { pacity monitor }\end{array}$ & $\begin{array}{l}\mathrm{dc} \text { and ac cur- } \\
\text { rent sensor } \\
\mathrm{ACS} 71230 \mathrm{~A}\end{array}$ \\
\hline 3 & Battery & $12 \mathrm{Ah}$ & $\begin{array}{c}\text { Motor and } \\
\text { inverter power } \\
\text { monitor }\end{array}$ & $\begin{array}{l}\text { dc voltage } \\
\text { sensor } \\
<25 \mathrm{~V}\end{array}$ \\
\hline 4 & Inverter & $100 \mathrm{~W}$ & $\begin{array}{l}\text { Monitor for } \\
\text { water level in } \\
\text { the water } \\
\text { storage }\end{array}$ & $\begin{array}{l}\text { ac voltage } \\
\text { sensor } \\
\text { zmpt101b }\end{array}$ \\
\hline 5 & Motor & $75 \mathrm{~W}$ & $\begin{array}{c}\text { ON/ OFF } \\
\text { Battery control }\end{array}$ & $\begin{array}{c}\text { Ultrasonic } \\
\text { sensor } \\
\text { Hc-sr04 } \\
\end{array}$ \\
\hline 6 & $\begin{array}{c}\text { Water } \\
\text { storage }\end{array}$ & $10.000 \mathrm{~L}$ & $\begin{array}{c}\text { ON/ OFF } \\
\text { motor control }\end{array}$ & $\begin{array}{c}\text { Driver } 8 \text { chan- } \\
\text { nels relay }\end{array}$ \\
\hline 7 & & & Alarm & \\
\hline 8 & & & Record data & \\
\hline
\end{tabular}

Thereafter, the result of SCADA system planning includes SCADA system tested result. The SCADA system test is intended as an indicator that the system is successfully designed, as the testing purpose is to determine the extent to which the SCADA system designed can be operated properly and meet the minimum standard. Accordingly, the SCADA system test is divided into three, namely the system test on the HMI including the display design, the parameters being monitored, the controlled SPP components and other supporting features. Secondly, system tests on the RTU include data acquisition for each sensor and component control on the SPP. Thirdly, system tests on communication lines include data processing, data reception and communication range. The aim is to find out the signal strength of the xbee module ($\mathrm{dBm})$ to the range $(\mathrm{m})$ distance for each data packet transmission which is then able to provide information about the distance limits that can be reached by the xbee module.

\subsection{Result of HMI test}

The purpose of HMI test is to evaluate and ensure that HMI has good performance in processing data both to record data, display monitored parameters, status of equipment condition, alarm indicator, and control the system. The purpose of this HMI test is to observe the success of HMI in processing data both to record data, display monitored parameters, status of equipment condition, monitor the ON / OFF alarm and control the system. Test results on HMI test on data records for this SCADA system are provided in Fig. 8 and Fig. 9. Based on the Fig. 8, PV data is recorded for 11 hours, which is from 6 am to 5 pm with cloudy sunny conditions with sampling frequency in $1 \mathrm{~s} /$ sampling. The PV data record indicates that the SCADA system is capable to record data well and is able to respond to changes in exposure conditions in PV.

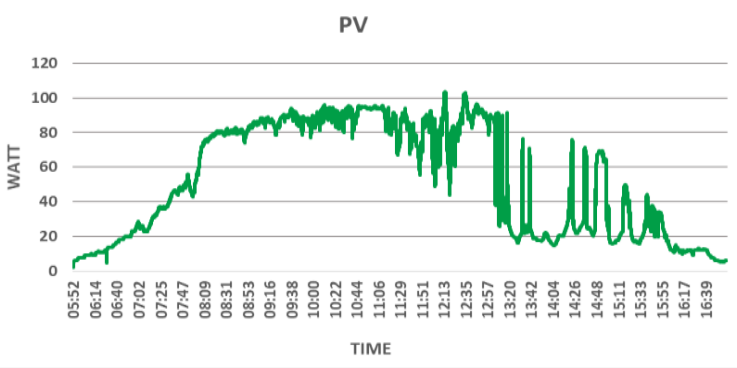

Fig. 8: Result of PV data record from 05.52 am to $17.00 \mathrm{pm}$ 
In addition, the Fig. 9 shows the battery data record, inverter, driver and water storage during water storage filling. The motor used is 25 watt with a water discharge of $16.6 \mathrm{~L} / \mathrm{min}$. The results indicate that the SCADA system is capable of recording data when extracting water to water storage on a scale of 0 to $100 \%$, recording a reduction in battery capacity due to being loaded, recording voltage stability of the output inverter and recording the amount of wattage used which is between 23 watt to 27 watt .

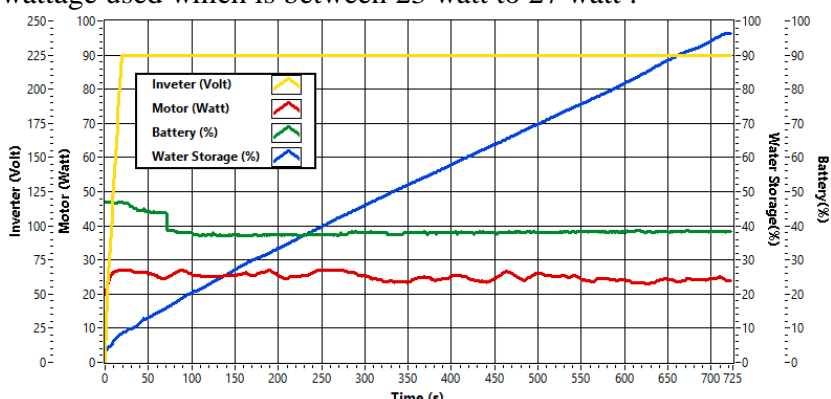

Fig. 9: Result of data record in battery, inverter, motor, and water storage

Fig. 10 provides the result of further test on display controlled parameters, equipment condition status, ON/ OFF alarm indicator, and control process. The HMI consists of six parts including PV, BCR, battery, inverter, motor, and water storage. The fig. 10 shows the PV that already designed. The figure also indicates that the HMI is able to process data with good performance.

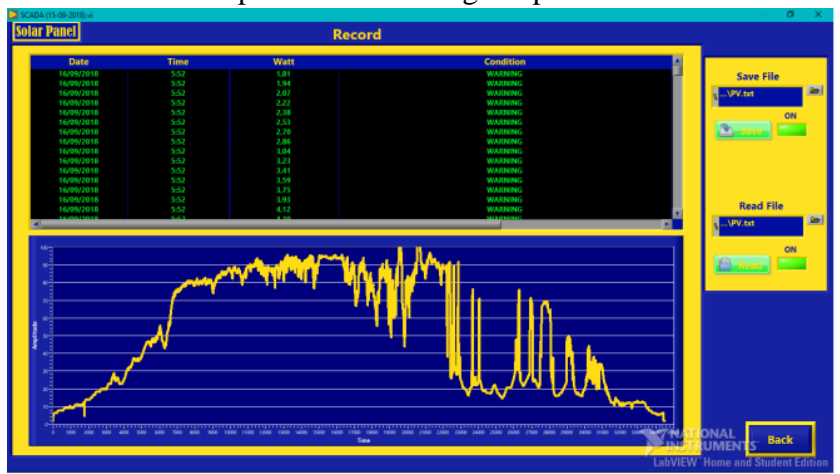

Fig. 10: Result of HMI test on PV section

\subsection{Result of RTU test}

The purpose of this test is to know the response of data acquisition for each sensor and the relay module's response in controlling the PLTS components. This test has two different test scenarios. The first scenario is monitoring the results of data acquisition from each sensor installed on the RTU, while the aim is to see the stability response of readings from sensors that have been used. The results of the first scenario testing can be seen in Fig. 11.

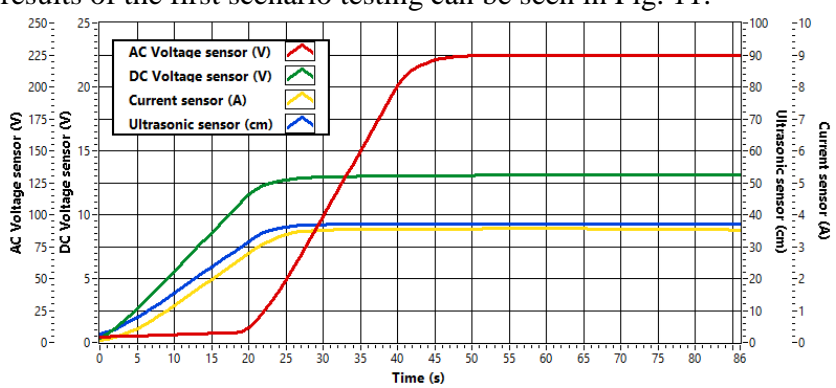

Fig. 11: Result of data acquisition in each sensor of SCADA system

The result of testing shows that RTU is capable of data acquisition in the form of the value of the magnitude of measurement through sensor that has been installed in the RTU. Stable reading value is obtained in 52 seconds from the initial reading of the working sensor. This is mainly caused by the filter applied in the sensor program. Applying this filter is in purpose to obtain the stable sensor reading value.

For the second scenario, RTU testing is conducted to controlling the relay module in the RTU. Observation is focused on the relay module's response to the ASCII character entered. The result of this second scenario is provided in Tab. 3 .

Table 3: Result of Relay Module Response Test

\begin{tabular}{|c|c|c|c|}
\hline No & Input ASCII & Relay & Condition \\
\hline 1 & A & Relay 1 & ON \\
\hline 2 & C & Relay 2 & ON \\
\hline 3 & E & Relay 3 & ON \\
\hline 4 & G & Relay 4 & ON \\
\hline 5 & B & Relay 1 & OFF \\
\hline 6 & D & Relay 2 & OFF \\
\hline 7 & F & Relay 3 & OFF \\
\hline 8 & H & Relay 4 & OFF \\
\hline
\end{tabular}

The result of testing shows that the RTU that is designed is able to respond to the control process that is carried out that is marked with the ON and OFF driver module installed in the RTU mentioned. If the input entered is ASCII characters, namely A, C, E or $\mathrm{G}$, the RTU responds to turn on the driver module. Then if the input entered is in the form of $\mathrm{B}, \mathrm{D}, \mathrm{F}$ or $\mathrm{H}$, the RTU responds to turn off the driver module.

\subsection{Result of communication line test}

The communication line test aims to determine the xbee module's signal strength $(\mathrm{dBm})$ to the range $(\mathrm{m})$ distance for each data packet delivery then observe the data packets sent with a percentage scale. In testing this communication line, the facilities used are provided or contained in Software X-ctu, namely the range test. In this test the interval time is set at $1000 \mathrm{~ms}$ intervals and the data sent is 100 times. The results of the communication line testing with a distance of $200 \mathrm{~m}$ can be seen in Fig. 12 .

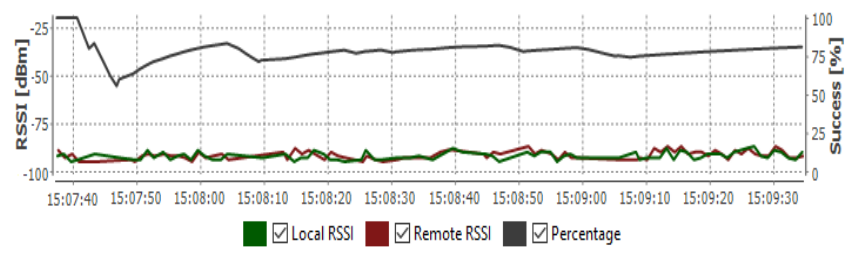

Fig. 12: Result of communication line test with $200 \mathrm{~m}$ distance

The result reported that longest distance of the xbee module with xbeeS2C series that is used is capable of transferring data as far as $200 \mathrm{~m}$ with $81 \%$ data received successfully and signal strength of $-92 \mathrm{dBm}$. Apart from that, testing is also carried out with a distance of $250 \mathrm{~m}$. the results of this test can be seen in Fig. 13.

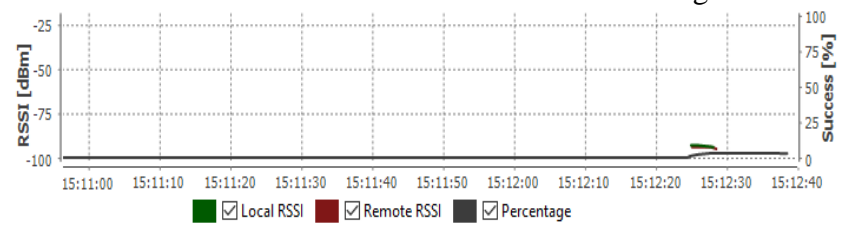

Fig. 13: Result of communication line at $250 \mathrm{~m}$ of distance

This testing result indicates that at distance of $250 \mathrm{~m}$, xbee module used is only able to transfer a percentage data received at $3 \%$ with signal strength of $-95 \mathrm{dBm}$. While Fig. 14 shows a communication line test result from various distance, ranging from 50 to $250 \mathrm{~m}$. 


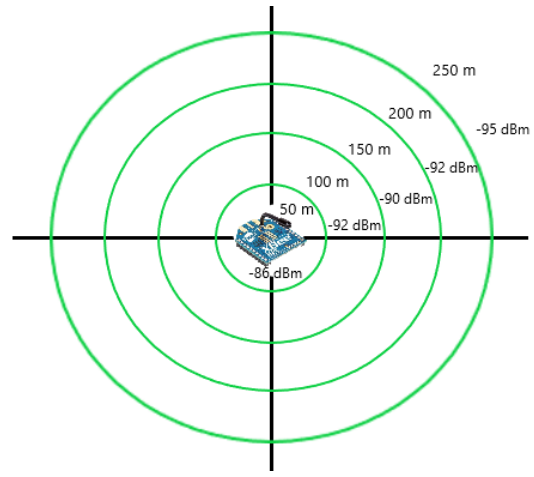

Fig. 14: Result of communication line test in various distance ranging from 50 to $250 \mathrm{~m}$

\section{Conclusion}

Planning of SCADA based solar power plant system in the clean water irrigation system is successfully processes the data including data record, display monitored parameters, status of equipment conditions, alarm indicator, and able to control the system efficiently. The RTU designed is also capable for data acquisition in the form of measurement quantities through sensors and able to respond to the control process carried out on the driver module installed on the RTU. The farthest distance of the xbee module range with the xbeeS2C series used is able to transfer data as far as $200 \mathrm{~m}$ with a percentage of data that has been successfully received by $81 \%$ and signal strength of $-92 \mathrm{dBm}$. As for the distance of $250 \mathrm{~m}$ the percentage of data successfully received is $3 \%$ and the signal strength value is $-95 \mathrm{dBm}$.

\section{Acknowledgement}

This research topic is strategic for supporting the renewable energy integration into the national grid, which the integration level is $23 \%$ in 2025 as mention in National Energy Policy.

We are gratefully acnowledge the funding from USAID through the SHERA program - Centre for Development of Sustainable Region (CDSR).

\section{References}

[1] D. Delimustafic, J. Islambegovic, A. Aksamovic, and S. Masic "Model of a hybrid renewable energy system: Control, supervision and energy distribution," in 2011 IEEE International Symposium on Industrial Electronics, 2011, pp. 1081-1086.

[2] A. Tsagaris and D. G. Triantafyllidis, "Data monitoring system for supervising the performance assessment of a photovoltaic park," in CINTI 2012 - 13th IEEE International Symposium on Computational Intelligence and Informatics, Proceedings, 2012, pp 385-389.

[3] S. Tiwari and R. N. Patel, "Real Time Monitoring of Solar Power Plant and Automatic Load Control," in Engineering and Systems (SCES), 2015 IEEE Students Conference on, 2015, pp. 1-6.

[4] P. Guerriero, F. Di Napoli, G. Vallone, V. Dalessandro, and S. Daliento, "Monitoring and diagnostics of PV plants by a wireless self-powered sensor for individual panels," in IEEE Journal of Photovoltaics, 2016, vol. 6, no. 1, pp. 286-294.

[5] P. Guerriero, G. Vallone, M. Primato, F. Di Napoli, L. Di Nardo, V. D'Alessandro, and S. Daliento, "A wireless sensor network for the monitoring of large PV plants," in 2014 International Symposium on Power Electronics, Electrical Drives, Automation and Motion, 2014, pp. 960-965.

[6] G. Bayrak, M. Cebeci, and x, "Monitoring a grid connected PV power generation system with labview," in IEEE Renewable Energy Research and Applications (ICRERA), 2013 International Conference on, 2013, no. October, pp. 562-567.

[7] C. I. Chow and J. Mungkornassawakul, "A smart recording power analyzer prototype Using LabVIEW and low-cost data acquisition (DAQ) in being a smart renewable monitoring system," in IEEE
Green Technologies Conference, 2013, pp. 49-56.

[8] C. Lin, G. Jie, Z. Wu, and W. Rui, "Design of networked monitoring system of PV grid-connected power plant," in Proceedings of 2011 International Conference on Electronic and Mechanical Engineering and Information Technology, EMEIT 2011, 2011, vol. 3, pp. 1169-1172.

[9] A. Soetedjo, Y. I. Nakhoda, and D. Suryadi, "Development of data acquisition system for hybrid power plant," in 2013 13th International Conference on Quality in Research, QiR 2013 - In Conjunction with the 2nd International Conference on Civic Space, ICCS 2013, 2013, pp. 197-201.

[10] E. Duque, A. Isaza, P. Ortiz, S. Chica, A. Lujan, and J. Molina, "Urban Sets Innovation: design of a solar tree PV system for charging mobile devices in medellin - Colombia," in 6th International Conference on Renewable Energy Research and Applications, 2017, pp. 495-498.

[11] I. Abubakar, S. N. Khalid, M. W. Mustafa, H. Shareef, and M. Mustapha, "Calibration of ZMPT101B voltage sensor module using polynomial regression for accurate load monitoring," in ARPN Journal of Engineering and Applied Sciences, 2017, vol. 12, no. 4, pp. 1076-1084.

[12] Allegro, "ACS712 Fully Integrated, Hall Effect-Based Linear Current Sensor with 2.1 kVRMS Voltage Isolation and a LowResistance Current Conductor,” 2007, Rev. 7., pp. 1-14. 